\title{
Simulation of Deep Turbulence Compensation for a Laser Phased Array
}

\author{
Jack E. McCrae, Jr. \\ Air Force Institute of Technology \\ Department of Engineering Physics \\ Center for Directed Energy (AFIT/CDE) \\ 2950 Hobson Way \\ Wright-Patterson AFB, OH 45433-7765 \\ and \\ Oak Ridge Institute for Science and Education, \\ 1299 Bethel Valley Road, Oak Ridge, TN, 37830 \\ 937-255-3636 x4739 \\ jack.mccrae.ctr@afit.edu
}

\author{
Steven T. Fiorino \\ Air Force Institute of Technology \\ Department of Engineering Physics \\ Center for Directed Energy (AFIT/CDE) \\ 2950 Hobson Way \\ Wright-Patterson AFB, OH 45433-7765 \\ 937-255-3636 x4506 \\ steven.fiorino@afit.edu
}

\begin{abstract}
The performance of a 127 element phased array laser system is simulated for the case of an extended target in the presence of strong atmospheric turbulence. The phases of the individual sub-apertures of a phased array laser system can be controlled to compensate for the beam quality degradation caused by atmospheric turbulence. This is done by setting the phases of the sub-apertures to the conjugate of the phase received from a beacon located at the target. As the level of turbulence increases, the ability of an adaptive optics system to correct for turbulence decreases; the quality of this compensation decreases due to increases in both scintillation and fitting error. In the case where the laser system itself is used to create the beacon on an extended target, the quality of the beacon produced is also degraded by this atmospheric turbulence and this further impacts the quality of the compensation. This study simulates a $10 \mathrm{~km}$ tactical engagement to quantify the performance impacts due to turbulence, and the portion of this impact due to the inability of the system to form a perfect beacon in these conditions. Performance is measured by both Strehl ratio and power delivered within a near diffraction limited circle. Results are presented comparing uncompensated, compensated with a perfect beacon, and compensated with simulated beacon cases as the level of turbulence increases.
\end{abstract}

\section{TABLE OF CONTENTS}

1. INTRODUCTION ................................................1

2. METHODOLOGY .........................................1

3. RESULTS..........................................................5

4. CONCLUSIONS .....................................................8

REFERENCES.....................................................10

BIOGRAPHIES..............................................10

ACKNOWLEDGEMENTS........................................10

\section{INTRODUCTION}

The ability of a phased laser array to compensate for atmospheric turbulence based upon backscatter from a rough extended target is demonstrated with simulations. Cases demonstrated include uncompensated propagation, beacon compensated propagation, and self-created beacon compensated propagation. A 127 element laser array with piston phase only control of the subapertures is used throughout.

The concept of using laser arrays for directed energy (DE) applications is enjoying some currency thanks to advances in electric laser technology in general and fiber laser technology in particular. Fiber laser phased array systems offer potential advantages in size, weight, efficiency, and scalability over more conventional designs, plus the promise of new capabilities and features such as electronic beam steering, distributed apertures, conformal apertures, and graceful degradation. [1,2,3,4]

The next sections lay out the simulation in detail, present example cases, and conclude with a discussion of the results obtained. The methodology section details the hypothetical physical parameters of the array simulated, the mapping of these parameters into the simulation space, and the construction and operation of the components of the simulation. The results section details examples of compensated and uncompensated propagations. The paper concludes with a discussion of these results and plans for future effort.

\section{Methodology}

After some preliminary definitions, implementation details and the techniques used for each part of the simulation will be presented. Then these pieces will be illustrated working together to produce example field distributions at the target plane.

\section{Definitions}

Atmospheric turbulence and its effects can be quantified with various tools, a few of these will be introduced now. The refractive index structure parameter constant, $C_{n}^{2}$, quantifies the local turbulence strength by scaling the spatial power spectrum of the refractive index. A modified von Kármán spectrum with a $1 \mathrm{~cm}$ inner scale and $100 \mathrm{~m}$ outer scale are used throughout this work. [5] The Fried parameter, $r_{0}$, also called the atmospheric coherence width, characterizes the lateral separation at which points in a light beam become poorly correlated. [6] Intensity variations 
across a laser beam can be characterized by the logamplitude variance, $\sigma_{\chi}^{2}$, of the light field. This quantity is computed just as it is named: the variance of the real-part of the logarithm of the complex-valued light field. For a spherical wave, $r_{0}$ and $\sigma_{\chi}^{2}$ can be computed from $C_{n}^{2}$ as [5]:

$$
\begin{aligned}
& r_{0, s w}=\left[0.423 k^{2} C_{n}^{2}(z)\left(\frac{z}{\Delta z}\right)^{5 / 3} d z\right]^{-3 / 5} \\
& \sigma_{\chi, s w}^{2}=0.563 k^{7 / 6} \int_{0}^{\Delta z} C_{n}^{2}(z) z^{5 / 6}\left(1-\frac{z}{\Delta z}\right)^{5 / 6} d z
\end{aligned}
$$

where $k=2 \pi / \lambda$. In these simulations, the wavelength, $\lambda$, is $1.07 \mu \mathrm{m}$ and $\Delta z$ is $10 \mathrm{~km}$. The spherical-wave versions of these relationships are appropriate here for both the laser beam, which we endeavor to focus on the target, and the light scattered from the target, which spreads widely as it travels back towards the source. The simulations conducted here used a constant level of turbulence along this path, so choosing any one of $C_{n}^{2}, r_{0}$, and $\sigma_{\chi}^{2}$ allows the other two to be computed. This work will mostly use $r_{0, \mathrm{sw}}$ to quantify the turbulence present.

Turbulence can be called deep when it is strong enough to cause substantial intensity variations or scintillation in a laser beam. One definition has the strong fluctuation regime as where the log-amplitude variance of the beam is much greater than $1 / 4$. [5] That is:

$$
\sigma_{\chi}^{2} \gg 0.25
$$

This requirement is adequately satisfied, perhaps incidentally, in this study where the goal was to investigate turbulence strong enough to interfere with the formation of a beacon at the target.

The performance measure used here in most places will be the Strehl ratio. The Strehl ratio is defined as the maximum intensity obtained at the target plane in a simulation divided by the maximum intensity that would have been obtained from a perfect source in the absence of turbulence. This measure might be more appropriately called the peak Strehl ratio, since ordinarily the Strehl ratio is based on the intensity measured on-axis. [5,6,7]

\section{Implementation details}

This effort studied a 127 element coherent laser array. The sub-apertures were arranged in a hexagonal pattern with each sub-aperture uniformly illuminated. The array simulated was sized to fit within a $32 \mathrm{~cm}$ circle with $30 \mathrm{~cm}$ devoted to aperture and $2 \mathrm{~cm}$ given to the gutters between elements across a long diagonal. This choice was made to keep the geometry similar to a 6 element array which was studied earlier. [4] In the present case, this results in $2.3 \mathrm{~cm}$ diameter sub-apertures placed on about $2.5 \mathrm{~cm}$ centers. The cases simulated here are all for a $10 \mathrm{~km}$ range, which is considered a tactically interesting distance, reasonably possible for a variety of engagements. A constant level of atmospheric turbulence was simulated between the array and target planes, and this level was adjusted to achieve the desired value for the Fried Parameter $\left(r_{0}\right)$ for each simulation run. The simulated laser wavelength was 1.07 $\mu \mathrm{m}$. Since non-linear effects like thermal blooming are not included in this simulation, the absolute amount of laser power simulated is meaningless; results are presented as proportional quantities.

To adequately sample this array and accurately and efficiently simulate the propagation, a 512 by 512 element grid with $2.5 \mathrm{~mm}$ sample spacing was chosen. This grid spacing was held constant along the propagation path for this simulations conducted here. The pixels of this grid are clearly visible on Fig. 1 which illustrates the laser array within the central region of this grid. The sub-apertures compute to be 9.2 samples wide, and as implemented the average sub-aperture contains 67 sample points, with a high of 69 and a low of 65 . The beam was propagated in $500 \mathrm{~m}$ steps from the source to the target plane, except the first and last steps which were $250 \mathrm{~m}$ each; this allowed the 20 phase screens used along the path to each be in the middle of a 500 $\mathrm{m}$ segment. The diffraction limit (R $\lambda / \mathrm{D})$ is $3.3 \mathrm{~cm}$ for this $10 \mathrm{~km}$ propagation (where D is taken as the $32 \mathrm{~cm}$ enclosing circle at the array plane, resulting in the smallest diffraction limit which is relevant here). The target plane is thus also well sampled on this grid.

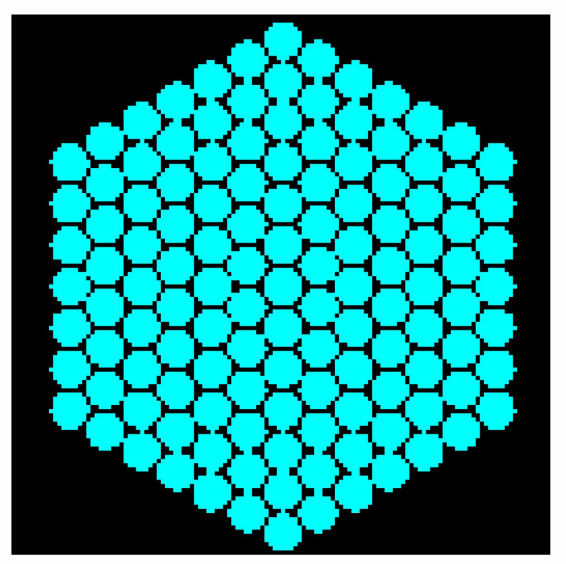

Figure 1 - The 127 element array as sampled within a $128 \times 128$ point grid, excised from the 512 x 512 point propagation grid.

An angular spectrum method Fresnel propagator was used to compute the laser field at each intermediate plane based upon the previous plane. [5] This method is also called the Fresnel Transfer Function Approach. [7] Each propagation consists of multiplications by phase terms and two fast Fourier Transforms (FFTs). To adequately sample the phase terms the propagation geometry needs to be arranged so:

$$
N \geq \frac{\pi \lambda \Delta z}{\delta^{2}}
$$


where $N$ is the number of samples on a side (512), $\lambda$ is the wavelength $(1.07 \mu \mathrm{m}), \Delta z$ is the propagation distance per step $(500 \mathrm{~m})$, and $\delta$ is the sample spacing $(2.5 \mathrm{~mm})$. For this simulation, the grid is over sampled, since the above equation recommends $\mathrm{N}$ be at least 269 and 512 points are used on a side. In fact 512 is the smallest power of two larger than 269, which helps speed up the computation of the FFTs. For the shorter partial propagations at each end of the path, this criterion shows the grid is even more well oversampled. The entire propagation however is mathematically equivalent to the 21 partial propagations used to create the entire path and thus the whole length would be seriously undersampled if nothing further were done. To prevent the aliasing which would be caused by this effect absorbing boundary conditions are used at each intermediate plane. A smooth taper based on a half cycle of a sinusoid is used to create these absorbing boundary conditions, starting one-eighth of the way from each edge and reaching zero on the edge. These simulations were created with Mathematica.

\section{Vacuum Propagation}

Further results will be compared to the diffraction-limited results of a vacuum propagation. This is obtained by turning off the turbulence, but otherwise using the same propagation code. The result from this propagation is shown in Fig. 2. These results show the grid used captures the far-field pattern well. The first set of sidelobes are captured nicely by this grid, although the absorbing boundary conditions are just starting to show their presence at this point. The absence of ringing on Fig. $2 b$ beyond the largest sidelobes is possibly due to these boundary conditions. When absorbing boundary conditions aren't used aliasing becomes quite apparent.

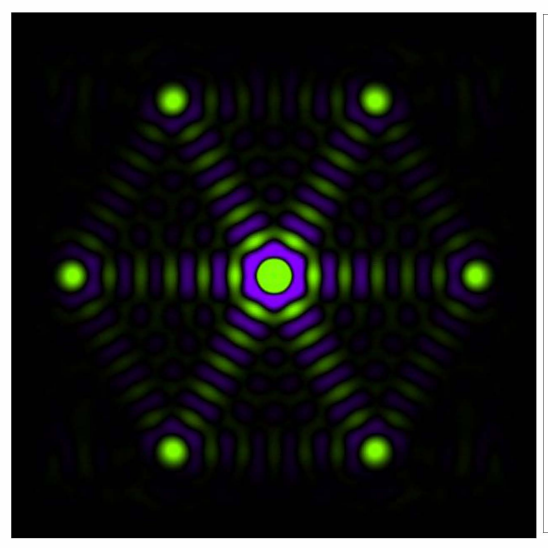

Figure 2 a

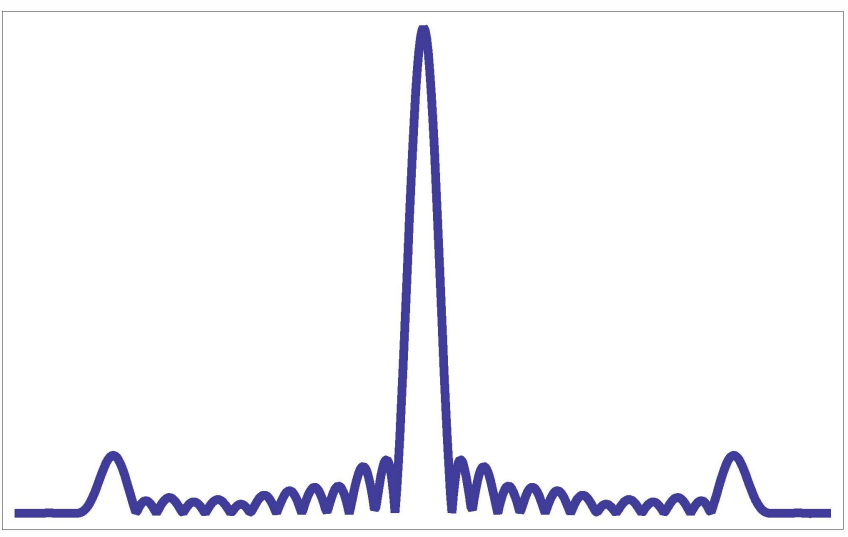

Figure 2 b

Figure 2 - (a) The diffraction limited far field amplitude from the 127 element array. Amplitude is shown as brightness and phase is displayed as hue. The brightness scale is clipped to enhance detail. Green and purple are 180 degrees out of phase. (b) The central horizontal slice through this data, now showing the absolute value of the electric field as height.

\section{Phase Screens}

Turbulence in the atmosphere distorts laser beams due to tiny differences in refractive index between different parcels of air. These differences are mostly attributed to small temperature changes. In this simulation, these refractive index variations are lumped into a discrete number of phase screens; the simulations discussed here use 20. These screens are generated by filtering complex Gaussian white noise to achieve screens with the desired refractive index power spectrum. This approach is far from perfect, and it is well known to get some relatively big things wrong, but these issues aren't of great importance to this simulation. Most particularly, this Fourier Transform approach doesn't give a statistically correct global tilt, since tilt involves spatial frequencies lower than one per total grid size, and one per total grid size is the lowest non-zero frequency a Fourier Transform contains. In fact, since the Fourier Transform approach gives results which wrap around smoothly, the global tilt on screens generated with this method is almost necessarily zero. While sub-harmonics could be added to make the phase screens used here more statistically correct, the tilt part raises the risk that the laser beam would be steered off the grid, necessitating a larger grid for little benefit gained. The power spectrum chosen for use here is the modified von Kármán power spectrum. [6] Example phase screens as used here are illustrated on Fig. 3.

\section{Simulating a Point Source}

Atmospheric turbulence may be compensated by using a beacon from the target to measure the effects of the turbulence and then apply the appropriate correction to the outgoing phased array beam. The ideal beacon would be a point source located at a selected aim-point on the target, and that is approximately what will be simulated here. A single pixel point source however would alias badly if propagated through this model and would not provide an accurate model of real results. A single pixel point source causes diffraction which spreads out fast enough to miss the 
baffles created by the absorbing boundary conditions. To deal with this issue, a simulated point source is created that has a finite spatial extent. By making the point source small enough that its diffraction limit still over fills the phased array, but not so large that light escapes from the edges of the grid, good results can be obtained. Since the point

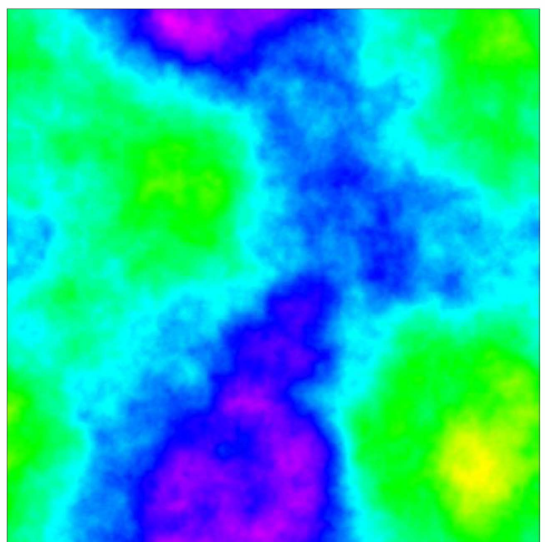

Figure 3 a

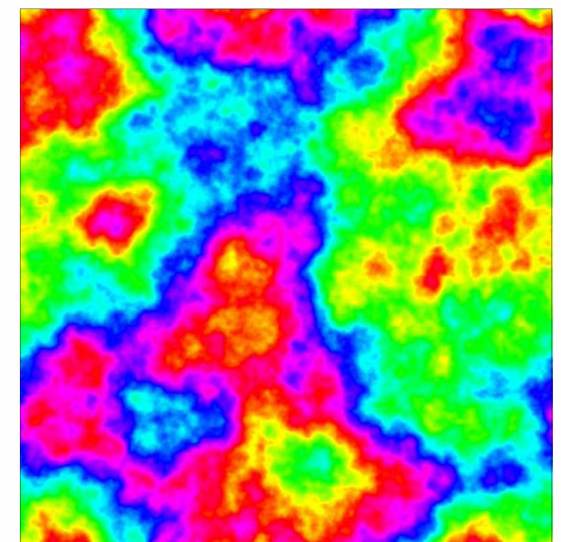

Figure 3 b

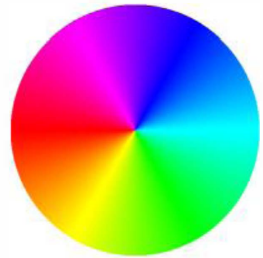

Figure 3 c

Figure 3 - (a) A random phase screen representing $500 \mathrm{~m}$ of atmospheric turbulence for a case where a $10 \mathrm{~km}$ propagation through 20 such screens would result in an $r_{0}$ of $10 \mathrm{~cm}$. (b) Same as Fig 3 a, except $r_{0}$ would now be $2.5 \mathrm{~cm}$. (c) The color wheel illustrating phase as hue. The phase at any point is given modulo $2 \pi$ by the angle from the center.

source's diffraction limit needs to over fill the telescope, this point source must be smaller than the array's diffraction limit, and thus it appears to be a point source to the phased array system. The point source simulator used here is a two dimensional sinc function (i.e. $\sin (\mathrm{x}) / \mathrm{x}$ ) multiplied by $\mathrm{a}$ Gaussian envelope and focused on the telescope (from the target). This produces, at least in the vacuum, a (nearly) uniformly illuminated square, well overfilling the telescope, and tapering to zero smoothly before reaching the absorbing boundary. The Gaussian envelope in the source plane becomes a convolution by a Gaussian at the far field destination plane and this provides the smooth roll-off at the edges. Fig. 4 illustrates the simulated point source and its field as vacuum propagated to the array plane. The sinc function was chosen to have a width of $1.7 \mathrm{~cm}$ from peak to its first zero (half the array diffraction limit), and the Gaussian envelope was chosen to have a standard deviation of $16 \mathrm{~cm}$ to provide good smoothing in both the telescope and target planes.

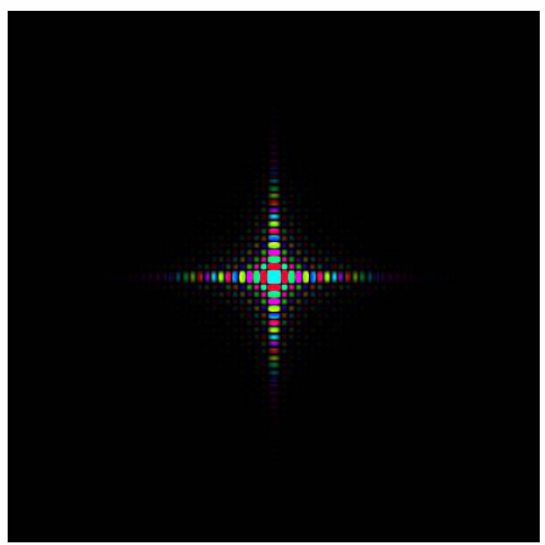

Figure 4 a

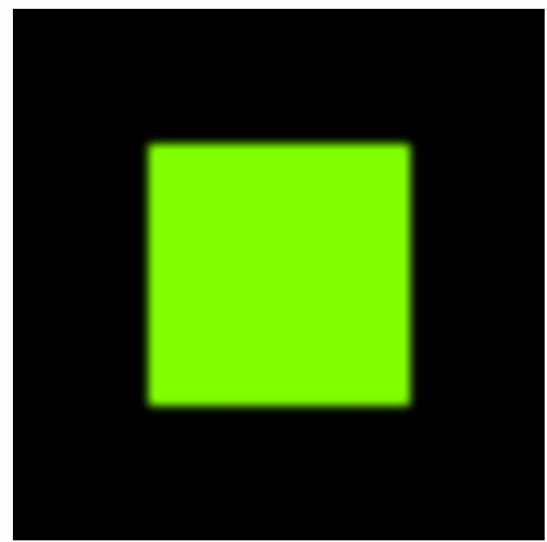

Figure 4 b

Figure 4 - (a) A simulated point source emanating from the target plane. This field is focused at the telescope plane. Sidelobes exaggerated due to brightness scale. (b) The field at the telescope plane propagated from the point source.

\section{Simulating Rough Surface Scatter}

Light scattered from a rough surface can be simulated by completely scrambling the phase of the field reflected from each point on the surface. If used alone, this approach encounters the same difficulty discussed above for a single pixel point source, namely severe aliasing. The solution is 
essentially the same as that given above as well, to replace each point on the surface with a patch of finite extent and to only propagate the portion of the light which is headed back to an area enclosing the laser array. Mathematically this is accomplished by first scrambling the phase of the scattered light then convolving this result with the point source given above, and then finally multiplying this result by a quadratic phase term to focus this scattered light onto the array plane. The point source used in the convolution is the same sinc Gaussian product described above, the phase term, which is also the same as in the previous section, needs to be added last to make the scatter from each part of the target plane overlap on the array plane. This procedure results in a speckled effective source plane at the target. This speckled pattern is the same as the image of the target plane a telescope would obtain if the telescope aperture were the size of the illuminated area at the array plane. Figure 5 shows the results from this procedure for the case where the 127 element array described above is scattered from a rough target.

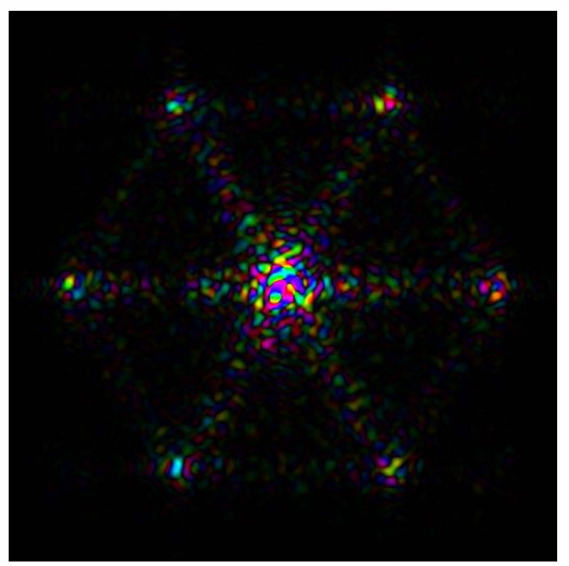

Figure 5 a

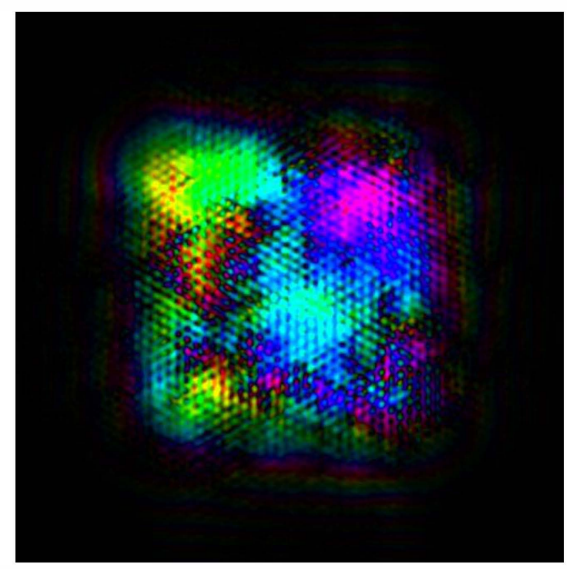

Figure 5 b

Figure 5 - (a) The speckled image of the array diffraction pattern at the target plane. (b) The field at the telescope plane propagated back from the rough surface target plane.

Fig. 5 demonstrates that this procedure appears to work as desired. The stippling pattern apparent on Fig. $5 \mathrm{~b}$ is due to interference between light coming from the sidelobes and the main lobe of the diffraction pattern at the target plane. It can be seen that speckle alone is enough to move some light out of the smooth square as seen in Fig. 4 b. The laser array itself is only as wide as a quarter of the whole grid, so it sits well inside the scattered light pattern seen in Fig. 5 b; this is as desired to prevent edge effects from corrupting the scatter which will be measured at the array and will be further discussed in the next section.

\section{RESULTS}

The simulations presented here compare four different scenarios for the same laser array. These cases are (1) uncompensated, (2) compensated with a perfect beacon, (3) one-pass compensated with a self created beacon, and (4) many-pass compensated with a self created beacon. At least one example for each of these cases is given in this section. Quite a few more cases need to be run to have statistical confidence in any trends observed.

\section{Uncompensated Propagation}

The first case is uncompensated propagation; the laser array is in phase at the source plane, and focused at the target plane. Fig. 6 shows the amplitude pattern obtained for a single instance of propagation through constant turbulence with the turbulence set so $r_{0, \mathrm{sw}}$ is $4.5 \mathrm{~cm}$, where $r_{0, \mathrm{sw}}$ is the value of $r_{0}$ which would be computed for spherical wave propagation through this turbulence. The spherical wave log-amplitude variance $\left(\sigma_{\chi, s w}^{2}\right)$ is computed to be 0.68 for these conditions. The value of $r_{0}$ for plane wave propagation, $r_{0, \mathrm{pw}}$, is $2.5 \mathrm{~cm}$ here, but this value is of little meaning in this simulation where the beams of interest are better considered as spherical waves emanating or focusing on the target.

\section{Compensated Propagation}

The second case is piston compensated propagation, presuming a perfect beacon. This procedure runs as follows: first, a simulated point source is propagated through discrete phase screens representing the desired turbulence to the telescope plane; at the telescope plane the average phase of the point source is computed over each sub-aperture; next, the conjugate of this computed average phase is applied to the sub-apertures, this amounts to correcting just the piston portion of the phase error on each sub-aperture; finally, this compensated field is propagated through the phase screens back to the target plane where the results can be studied. An example of this case is shown on Fig. 7. This case is examined in greater detail in the literature. $[8,9,10]$ 


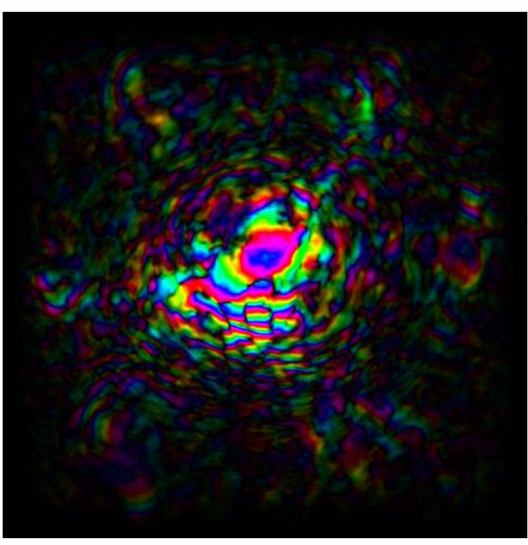

Figure 6 a

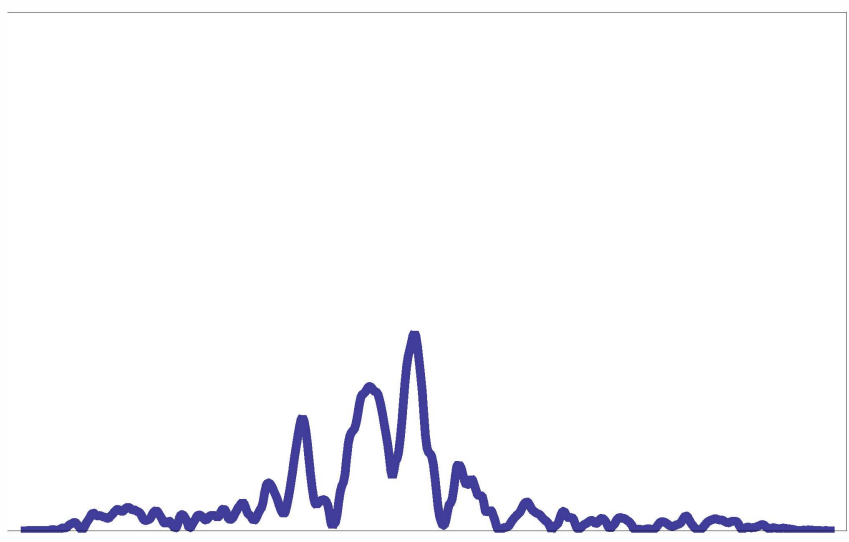

Figure 6 b

Figure 6 - (a) Example far field amplitude from the 127 element array for uncompensated case with $\boldsymbol{r}_{0, \mathrm{sw}}$ set to $4.5 \mathrm{~cm}$. (b) Horizontal slice through amplitude pattern capturing the peak value. Same scale as Fig. 2 b. The peak amplitude is only $38 \%$ of the vacuum case, and the peak intensity, or peak Strehl ratio, is only $15 \%$ of the vacuum case.

\section{One-Pass Self Compensated Propagation}

The third case is one-pass piston compensated propagation, using a self created beacon. This case is computed as follows: first, the array is focused at the target and propagated through the turbulence to the target plane. The field at the target plane would be the same as the example given as Fig 6. This field is then scattered from the presumed rough target as described above in section 2.5. This scattered field is propagated back through the phase screens to the telescope plane. Back at the telescope plane, this case now proceeds as the compensated case. The average phase is computed over each sub-aperture and the conjugate of this is applied to the sub-apertures and retransmitted to the target. An example of this case is shown on Fig. 8. It is typically the case that this one-pass compensation technique performs more poorly than the uncompensated case.

The rough surface scattering alone is enough to degrade the performance of this technique. Fig. 9 shows an example of results for this case with no turbulence. Speckle in the return is responsible for all the degradation observed in the compensated beam. For this particular example, it appears that the speckled return placed a branch point near the center of the array, and this resulted in the branch point (circulatory phase) seen inside the main lobe on Fig. 8 a. Running this case twice more didn't happen to reproduce such a result.

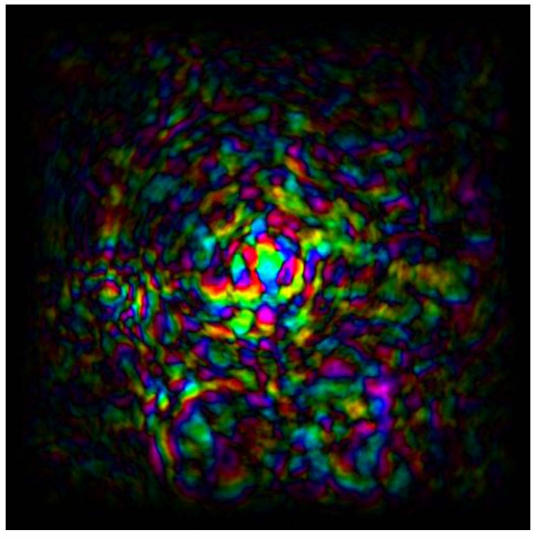

Figure 7 a

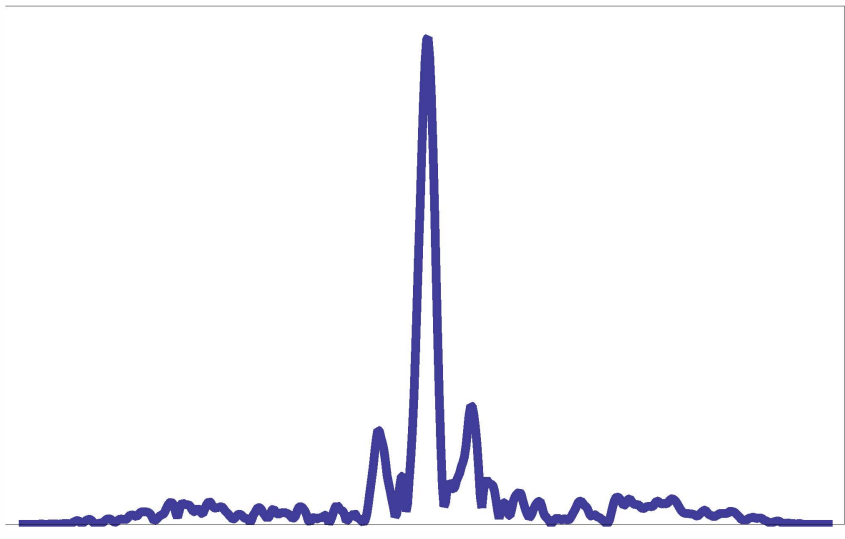

Figure 7 b

Figure 7 - (a) Example far field amplitude from the 127 element array for compensation based upon a perfect beacon with $r_{0, \mathrm{sw}}$ set to $4.5 \mathrm{~cm}$. (b) Horizontal slice through amplitude pattern capturing the peak value. Same scale as Fig. 2 b. The peak amplitude is $93 \%$ of the vacuum case, and the peak intensity, or peak Strehl ratio, is $88 \%$ of the vacuum case. 


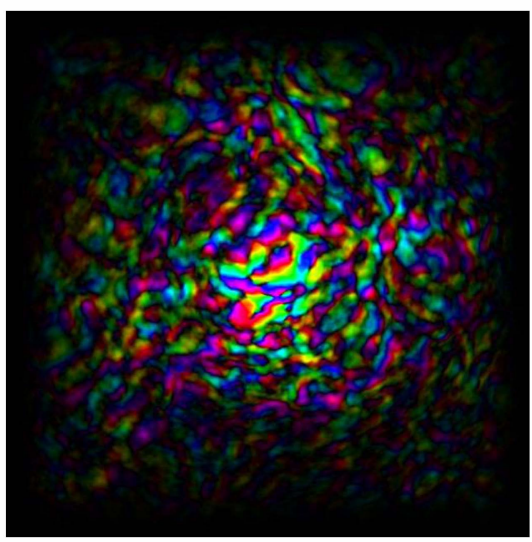

Figure 8 a

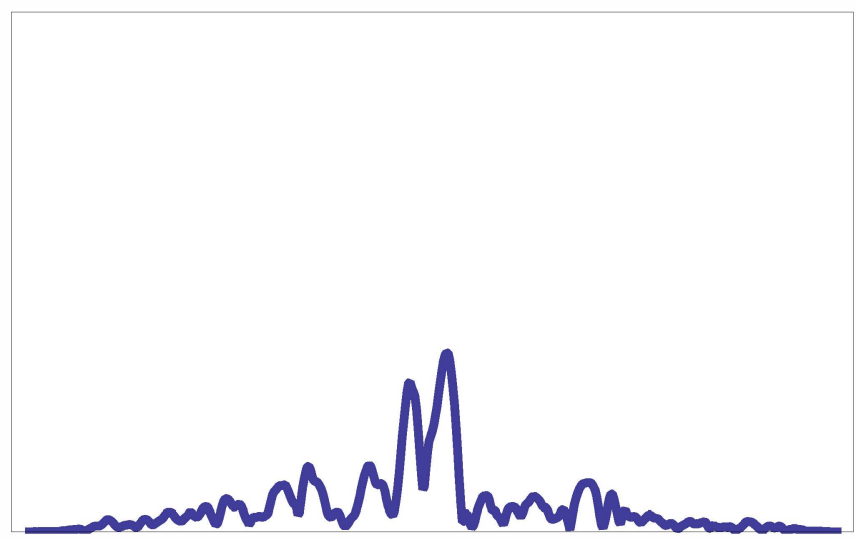

Figure 8 b

Figure 8. (a) Example far field amplitude from the 127 element array for one-pass compensation based upon a self created beacon with $r_{0, \mathrm{sw}}$ set to $4.5 \mathrm{~cm}$. (b) Horizontal slice through amplitude pattern capturing the peak value. Same scale as Fig. 2 b. The peak amplitude is only $34 \%$ of the vacuum case, and the peak intensity, or peak Strehl ratio, is only $12 \%$ of the vacuum case.

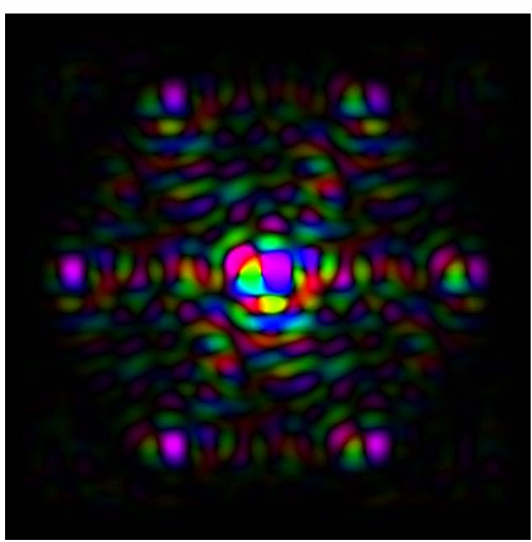

Figure 9 a

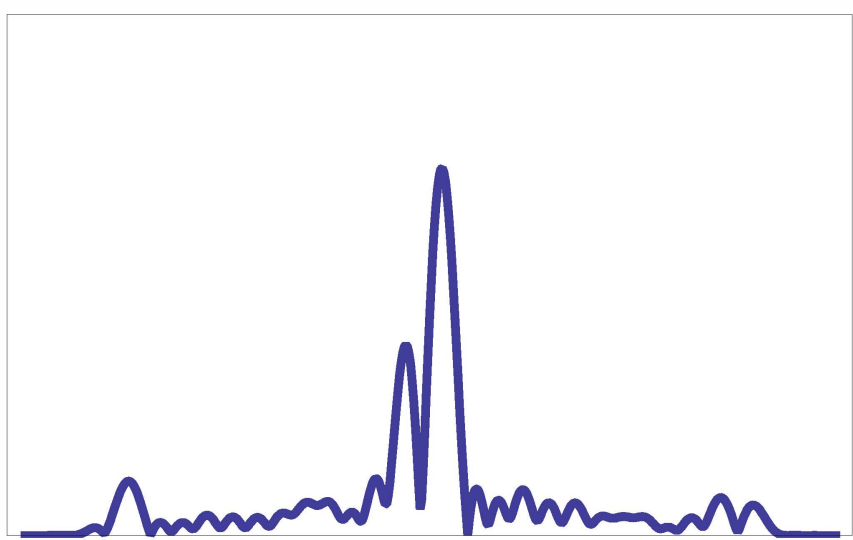

Figure 9 b

Figure 9 - (a) Example far field amplitude from the 127 element array for one-pass compensation based upon a self created beacon with $\boldsymbol{r}_{0}$ set to infinity. (b) Horizontal slice through amplitude pattern capturing the peak value. Same scale as Fig. 2 b. The peak amplitude is $70 \%$ of the vacuum uncompensated case, and the peak intensity, or peak Strehl ratio, is $50 \%$ of the vacuum uncompensated case.

\section{Multi-Pass SelfCompensated Propagation}

The final case is multi-pass piston compensated propagation, using a self created beacon. In this case the scattered field from one iteration of the procedure given above is used as the beacon to re-compensate the beam and this process is continued until convergence or an arbitrary cycle limit is hit. Fig. 10 illustrates an example of this case. It is typically the case here that the peak value in the field drifts away from the center of the array; that is quite apparent on Fig. 10 in this example. It is also typical the peak Strehl ratio is comparable to the result obtained with a perfect beacon, at least with the level of turbulence simulated here. It must be noted that the limited fidelity of the phase screen generator used, as discussed in the phase screens section above, make it likely the peak shifts seen here underestimate the truth. Fig. 11 shows a comparison of the intensity on target between the first uncompensated beam transmitted to the target and the final result after 100 iterations. The results aren't changing much between iterations at this point. The shift of the beam away from the center is quite apparent on both these plots.

Fig. 12 shows the Strehl Ratio as a function of iteration number for the example case above. Usually this procedure converges within 20 cycles or so for the turbulence level used here. Oscillation like that seen on the right of Fig. 11 is almost always present in some form, and some degree of overshoot (as seen near iteration 7) is often present as well. 


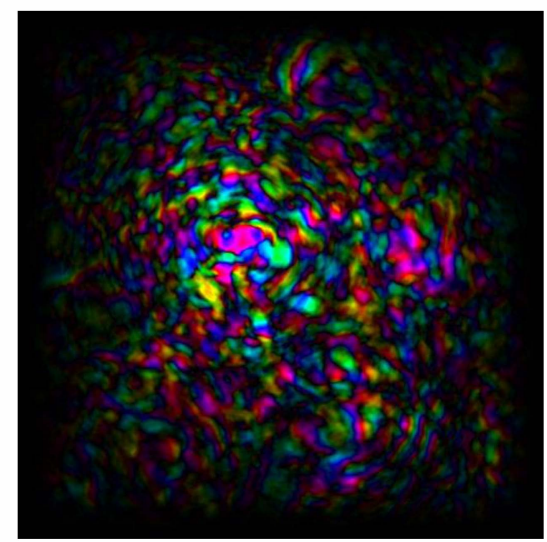

Figure 10 a

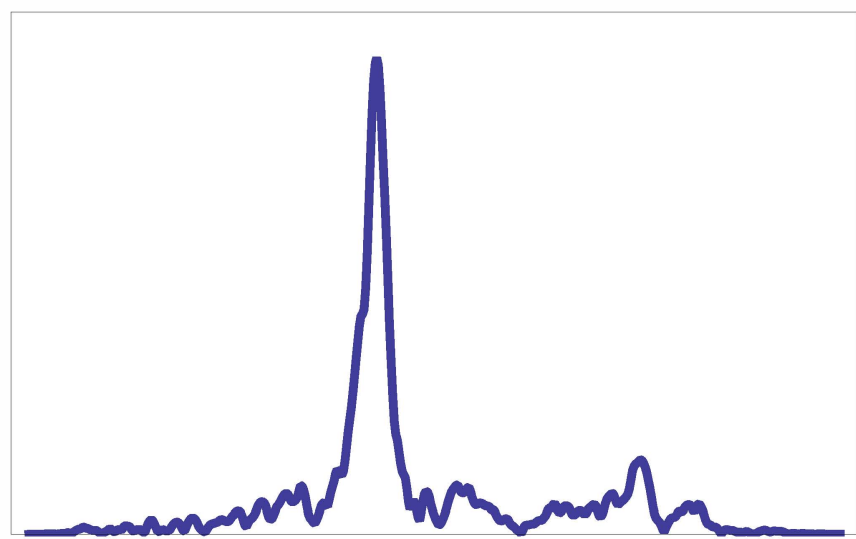

Figure 10 b

Figure 10 - (a) Example far field amplitude from the 127 element array for multi-pass compensation based upon a self created beacon with $r_{0}$ set to $4.5 \mathrm{~cm}$ after 100 iterations. (b) Horizontal slice through amplitude pattern capturing the peak value. Same scale as Fig. $2 \mathrm{~b}$. The peak amplitude is $91 \%$ of the vacuum case, and the peak intensity, or peak Strehl ratio, is $83 \%$ of the vacuum case.

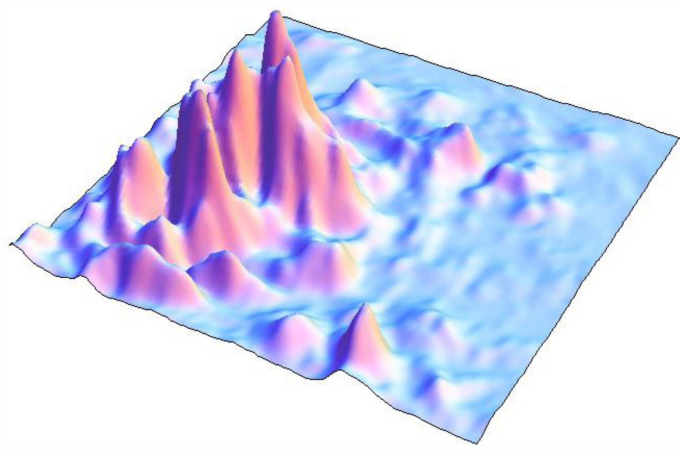

Figure 11 a

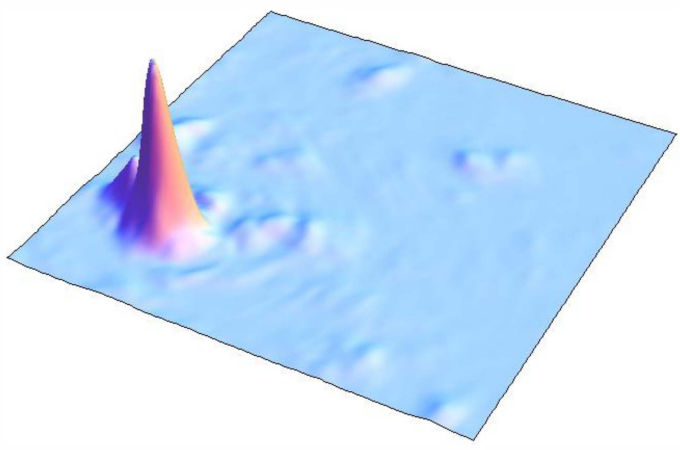

Figure 11 b

Figure 11 - Intensity as height for the central 128 by 128 sample region of the 512 by 512 computation grid. (a) Example far field intensity from the 127 element array for uncompensated transmission with $\boldsymbol{r}_{0, \mathrm{sw}}$ set to $4.5 \mathrm{~cm}$. (b) Example far field intensity after 100 iterations from the 127 element array for multi-pass compensation based upon a self created beacon with $r_{0, \mathrm{sw}}$ set to $4.5 \mathrm{~cm}$.

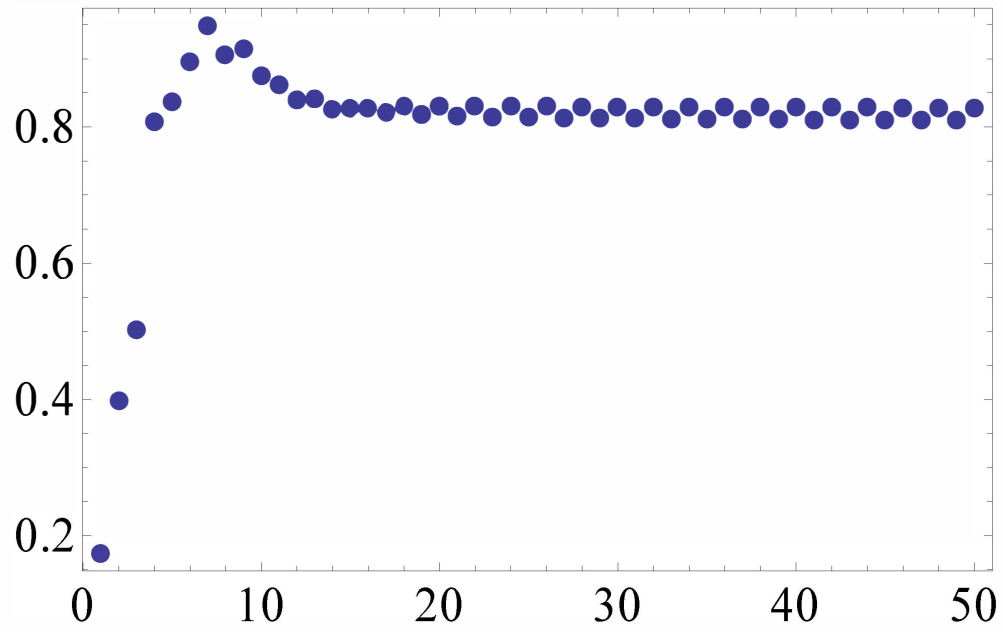

Figure 12 - Strehl Ratio vs. Iteration number for the example pictured in Fig. 10. 


\section{Conclusions}

\section{Discussion of results}

It is seen in the results presented above that the laser system as simulated here is capable of creating a beacon, and using this beacon to increase the intensity delivered on target. To further explore this approach, the level of turbulence was raised so $r_{0, s w}$ decreased to $2.5 \mathrm{~cm}$ then 200 separate cases were run for 50 iterations each. At this level of turbulence the log-amplitude variance $\left(\sigma_{\chi}^{2}\right)$ was 1.80 which is indicative of fairly deep turbulence. Fig 13 shows the average Strehl Ratio over these 200 cases at each iteration of beacon compensation. While substantial ringing was observed on many of the individual cases, often far greater than seen on Fig. 12, the average is much smoother, and while some of the individual cases appeared poorly converged after 50 iterations, the average result appears quite good.

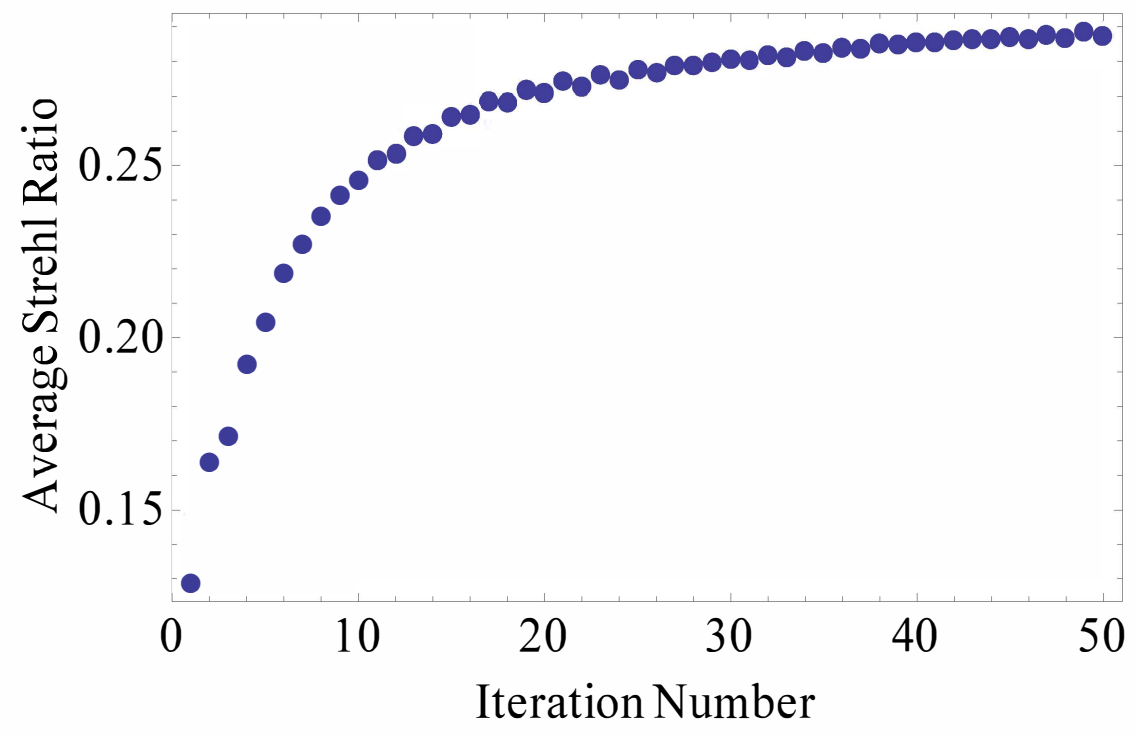

Figure 13 - Strehl Ratio vs. Iteration number for the average of 200 cases with $\boldsymbol{r}_{0, \mathrm{sw}} \mathrm{set}$ to $4.5 \mathrm{~cm}$.

Looking at the improvement of individual cases, little trend is seen. Fig. 14 is a scatter plot comparing the initial uncompensated Strehl Ratio and the final Strehl Ratio (after 50 iterations) for each of the 200 realizations simulated. The initial average Strehl Ratio was 0.133 , and the final average Strehl Ratio was 0.287, meaning that this approach more than doubled the peak on-target intensity. After just one iteration of this procedure, the average Strehl Ratio was 0.129 , just slightly below the uncompensated case.

\section{Future Work}

This effort suggests many directions for future research. The 200 cases simulated here is a single design point, and it would be interesting to vary a number of the parameters involved. The value of this technique as a function of the degree of turbulence is one of the first topics planned for further exploration. Also, a constant level of turbulence along the path was simulated here, and it would be valuable to change this so $r_{0}$ and $\sigma_{\chi}{ }^{2}$ could be varied independently. Other possible next-steps include limiting the size of the target surface, allowing the target surface, or the atmosphere to vary with time, and adding sub-aperture tilt compensation to the simulation. The overshoot on Fig. 12 is very interesting, and appears to be worth deeper exploration. The number of cases studied here is quite small, and running many more cases to better quantify the utility of this technique is an easy next step.

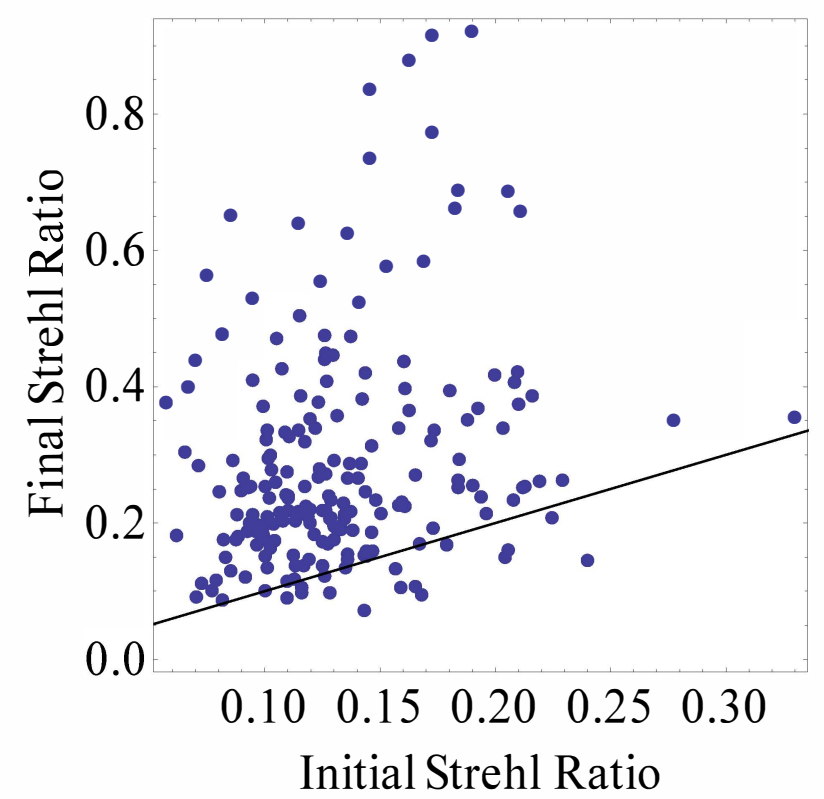

Figure 14 - Initial Strehl Ratio vs. Final Strehl Ratio for 200 cases with $r_{0, \mathrm{sw}}$ set to $2.5 \mathrm{~cm}$. The straight line is equal Strehl Ratios, so points above the line represent improvements, and those below the line are cases where compensation made the Strehl Ratio worse. 


\section{REFERENCES}

[1] Motes, R. A. and Berdine R. W., [Introduction to HighPower Fiber Lasers], Directed Energy Professional Society, Albuquerque, New Mexico, 1-12 (2009).

[2] Brignon, A. (Ed.), [Coherent Laser Beam Combining], Wiley, (2013)

[3] McManamon, P. F. and Thompson, W., "Phased array of phased arrays (PAPA) laser systems architecture," Fiber and Integrated Optics 22(2), 79-88 (2003).

[4] Van Zandt, N. R., Cusumano, S. J., Bartell, R. J., Basu S., McCrae, J. E., Fiorino, S. T., "Comparison of coherent and incoherent laser beam combination for tactical engagements", Opt. Eng. 51(10), 104301 (2012).

[5] Schmidt, J. D., [Numerical Simulation of Optical Wave Propagation], SPIE Press, Bellingham, Washington, (2010).

[6] Andrews, L. C. and Phillips, R. L., [Laser Beam Propagation Through Random Media], $2^{\text {nd }}$ Ed. SPIE Press, Bellingham, Washington, (2005).

[7] Voelz, D., [Computational Fourier Optics], SPIE Press, Bellingham, Washington, (2010)

[8] McCrae, J.E., N.R. Van Zandt, S.J. Cusumano and S.T. Fiorino, "Simulation of atmospheric turbulence compensation through piston only phase control of a laser phased array," (Proc. of SPIE Vol. 8733 873311) SPIE Defense, Security and Sensing Symposium, Baltimore, MD, 29 April - 2 May 2013.

[9] Vorontsov, M. A. and Lachinova, S. L. , "Laser beam projection with adaptive array of fiber collimators. I. Basic considerations for analysis", J. Opt. Soc. Am. A, 25(8), 1949 (2008).

[10] Lachinova, S. L. and Vorontsov, M. A., "Laser beam projection with adaptive array of fiber collimators. II. Analysis of atmospheric compensation efficiency", J. Opt. Soc. Am. A, 25(8), 1960 (2008).

\section{BIOGRAPHIES}

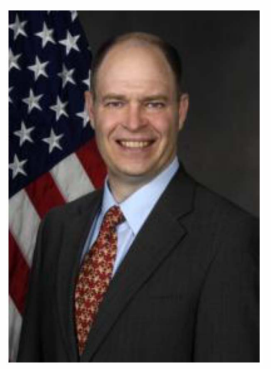

Jack McCrae received his Ph.D. in Physics from the Air Force Institute of Technology in 1997, an M.S. in Physics (Optics) from the Air Force Institute of Technology in 1993, and a B.S. in Physics from the Massachusetts Institute of Technology in 1984.

He is a retired Air Force Colonel with 27 years of service and currently a Senior Research Associate with the Center for Directed Energy at AFIT. His research interests include optics, lasers, quantum and non-linear optics, laser radar, atmospheric propagation and imaging.

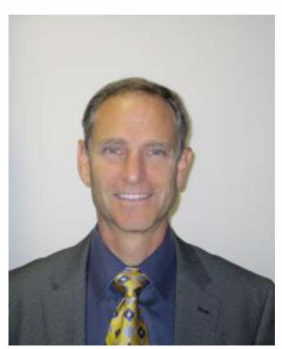

Steven T. Fiorino received his $B S$ degrees in geography and meteorology from Ohio State (1987) and Florida State (1989) universities. He additionally holds an $M S$ in atmospheric dynamics from Ohio State (1993) and a PhD in physical meteorology from Florida State (2002). He is a retired Air Force Lieutenant Colonel with 21 years of service and currently a research associate professor of atmospheric physics within the Engineering Physics Department at AFIT and is the director of the Center for Directed Energy. His research interests include microwave remote sensing, development of weather signal processing algorithms, and atmospheric effects on military systems such as high-energy lasers and weapons of mass destruction. Dr. Fiorino is a member of SPIE, AMS, AIAA and DEPS.

\section{ACKNOWLEDGEMENTS}

This work was funded in part by a grant from the High Energy Laser Joint Technology Office (HEL JTO) and the Air Force Office of Scientific Research (AFOSR). This research was supported in part by an appointment at the Air Force Institute of Technology administered by the Oak Ridge Institute for Science and Education through an interagency agreement between the U.S. Department of Energy and AFIT. The views expressed in this paper are those of the authors and do not necessarily reflect the official policy or position of the Air Force, the Department of Defense, or the U. S. Government. 